\title{
3D Mammary Colony-Forming Cell Assay
}

\author{
Giusy Tornillo ${ }^{1^{*}}$ and Sara Cabodi ${ }^{2}$
}

${ }^{1}$ Cardiff School of Biosciences, European Cancer Stem Cell Research Institute, Cardiff University, Cardiff, UK; ${ }^{2}$ Department of Molecular Biotechnology and Health Sciences, University of Turin, Turin, Italy

*For correspondence: TornilloG@cardiff.ac.uk

[Abstract] The mammary epithelium consists of multiple phenotypically and functionally distinct cell populations, which are organized as a hierarchy of stem cells, progenitors and terminally differentiated cells.

Identification of the mechanisms regulating the growth and differentiation of mammary stem and progenitor cells is of great interest not only to better understand the mammary gland development but also to clarify the origins of breast cancer, as these cells seem to be the likely targets of malignant transformation within the mammary epithelium. Hence, a variety of approaches have been developed for quantifying and studying these specific mammary cell subsets.

Given their high proliferative capacity, mammary progenitor cells are able to form colonies in vitro in low-density cultures. Here we describe how to perform a three dimensional (3D) Mammary Colony-Forming Cell (Ma-CFC) Assay, an in vitro functional assay suitable for the detection and analysis of mammary progenitor cells in feeder-free culture conditions.

Briefly, this protocol involves the seeding of mammary single cells, at clonal density, onto a semi-solid matrix (Matrigel), thus allowing mammary progenitors to proliferate and give rise to discrete 3D colonies. The number and the cell composition of the resulting colonies will vary according to the frequency and the differentiation potential of the progenitors, respectively.

\section{Materials and Reagents}

1. Single-cell suspension of primary mouse mammary cells (see Reference 1 for details about the dissociation of mouse mammary glands into single cells)

2. EpiCult ${ }^{\circledR}-B$ Basal Medium Mouse (STEMCELL Technologies, catalog number: 05611)

3. EpiCult ${ }^{\circledR}-\mathrm{B}$ Proliferation Supplements (STEMCELL Technologies, catalog number: 05612)

4. Trypan blue

5. Recombinant human Epidermal Growth Factor (EGF) (Sigma-Aldrich, catalog number: E9644) 
6. Recombinant human basic Fibroblast Growth Factor (bFGF) (Life Technologies, catalog number: PHG0023)

7. Heparin sodium salt (Sigma-Aldrich, catalog number: H3149)

8. Fetal Bovine Serum (FBS) (heat-inactivated) (Life Technologies, catalog number: 10270106)

9. Penicillin-Streptomycin (Pen/Strep) (Life Technologies, catalog number: 15070-063) Growth Factor Reduced (GFR) BD Matrigel ${ }^{\mathrm{TM}}$ Matrix [protein concentration $>8 \mathrm{mg} / \mathrm{ml}$, endotoxin levels < 2 Endotoxin Units (EU)/ml] (BD Biosciences, catalog number: 354230) Note: $B D$ Matrige $I^{T M}$ Matrix is supplied as a frozen solution. Thaw it on ice overnight and store as $1 \mathrm{ml}$ aliquots at $-20^{\circ} \mathrm{C}$. Leave Matrigel aliquots to thaw on ice for $2 \mathrm{~h}$ before use.

10. Complete EpiCult-B Medium (see Recipes)

\section{Equipment}

1. BD Falcon 8-well culture slides (BD Biosciences, catalog number: 354108)

2. Refrigerated centrifuge with swinging bucket rotor

3. Humidified $37^{\circ} \mathrm{C}, 5 \% \mathrm{CO}_{2}$ cell culture incubator

4. Inverted tissue culture microscope

5. Zeiss Observer Z.1 microscope (5x/0.12 objective)

\section{Software}

1. AxioVision Rel 4.8 software

2. Image $\mathrm{J}$

\section{Procedure}

1. The 8-well chamber slide is placed on ice and loaded with $80 \mu \mathrm{l}$ of Matrigel per well. The Matrigel drop is positioned in the centre of each well, taking care to avoid air-bubble formation.

Note: To prevent gelation, it is recommended to handle Matrigel on ice.

2. Next, the chamber slide is secured onto a pre-cooled adapter for swing-bucket rotor and centrifuged at $300 \times g$ (with a slow acceleration ramp) for $10 \mathrm{~min}$ at $4{ }^{\circ} \mathrm{C}$. The Matrigel layer is now flat and uniformly distributed onto the bottom of the chamber wells.

Note: It is convenient to use some plastic paraffin film to keep the lid of the culture slide in place during step 2. 
3. The chamber slide is then transferred to a cell culture incubator to allow Matrigel solidification for at least $15 \mathrm{~min}$.

4. Meanwhile, freshly prepared single mouse mammary cells are resuspended in Complete EpiCult-B Medium and counted.

Note: Trypan Blue stain is one of several methods recommended for viable cell counting.

5. The cell suspension is diluted in Complete EpiCult-B Medium to obtain a final concentration of 25,000 viable cells $/ \mathrm{ml}$.

6. A stock of Complete EpiCult-B Medium supplemented with $5 \%$ Matrigel is prepared by adding $50 \mu \mathrm{l}$ of Matrigel per $\mathrm{ml}$ of Complete EpiCult-B Medium and mixing by pipetting repeatedly.

7. The cell suspension from step 5 is mixed with the medium from step 6 in a 1:1 ratio. 400 $\mu \mathrm{l}$ of this mixture, which correspond to 5,000 cells in Complete EpiCult-B Medium 2.5\% Matrigel, are gently plated per well onto the solidified Matrigel from step 3 (Figure 1).

8. The chamber slide is placed in the cell culture incubator. The cells will settle onto the solid Matrigel layer.

9. The overlay liquid medium is replaced with fresh Complete EpiCult-B Medium supplemented with 2.5\% Matrigel every 3 days. To avoid damaging the bottom gel layer, the medium is aspirated from the corner of each well and the fresh medium is gently dispensed along the walls.

10. Colonies will be generated after 10-14 days of culture. Representative images are shown in Figure 2.

11. The number of colonies can be determined, using an inverted microscope, either by manual counting or with the support of image analysis programs, such as Image $\mathrm{J}$ software.

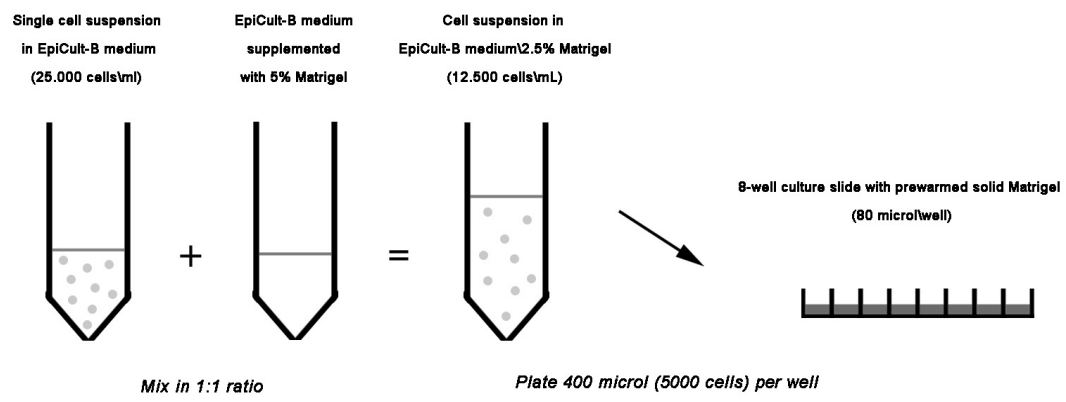

Figure 1. Schematic diagram depicting the procedure described above as step 7 


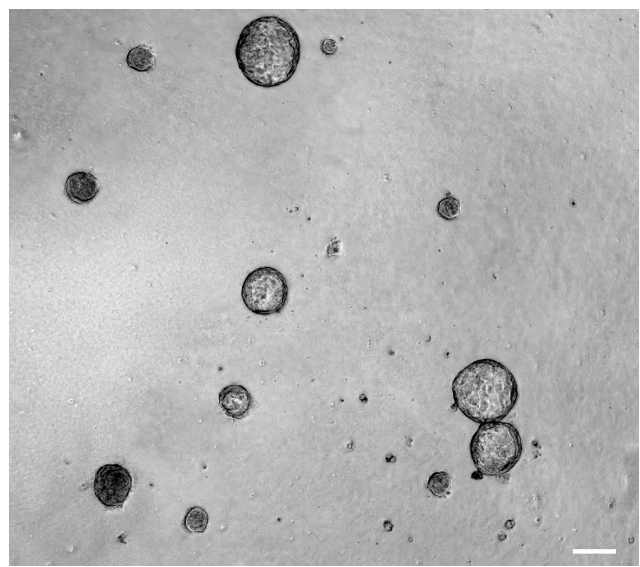

Figure 2. 3D mammary colony-forming cell assay. Phase-contrast image of colonies derived from 3D culture (day 10) of mouse mammary single cells. This image was obtained by using a Zeiss Observer Z.1 microscope (5x/0.12 objective) and the AxioVision Rel 4.8 software. Scale bar, $100 \mu \mathrm{m}$

\section{$\underline{\text { Recipes }}$}

1. Complete EpiCult-B Medium (50 ml)

EpiCult-B Basal Medium: $42 \mathrm{ml}$

EpiCult ${ }^{\circledR}-B$ Proliferation Supplements: $5 \mathrm{ml}$

FBS: $2.5 \mathrm{ml}$

Pen/Strep: $0.5 \mathrm{ml}$

EGF $(0.2 \mathrm{mg} / \mathrm{ml}): 2.5 \mu \mathrm{l}$

bFGF $(0.2 \mathrm{mg} / \mathrm{ml}): 2.5 \mu \mathrm{l}$

Heparin $(50 \mathrm{mg} / \mathrm{ml}): 4 \mu \mathrm{l}$

Filter-sterilize through a $0.2 \mu \mathrm{m}$ filter

Stored at $4{ }^{\circ} \mathrm{C}$ and use within one week

Complete EpiCult-B Medium must be supplemented with Matrigel immediately before use

\section{Acknowledgments}

We thank Dr Agata Tinnirello and Prof Senthil Muthuswamy for technical advice on the 3D overlay cell-culture method (previously described by Debnath et al. (2003). This work was funded by AIRC (Grant IG2008 and IG2011), MIUR (FIRB Giovani 2008), PiSTEM and Progetto Sanità Finalizzata. G. Tornillo was supported by a FIRC fellowship. 


\section{$\underline{\text { References }}$}

1. Debnath, J., Muthuswamy, S. K. and Brugge, J. S. (2003). Morphogenesis and oncogenesis of MCF-10A mammary epithelial acini grown in three-dimensional basement membrane cultures. Methods 30(3): 256-268.

2. Tornillo, G., Elia, A. R., Castellano, I., Spadaro, M., Bernabei, P., Bisaro, B., CamachoLeal Mdel, P., Pincini, A., Provero, P., Sapino, A., Turco, E., Defilippi, P. and Cabodi, S. (2013). p130Cas alters the differentiation potential of mammary luminal progenitors by deregulating c-Kit activity. Stem Cells 31(7): 1422-1433. 